\title{
Evaluating the cytotoxicity of contact lens multi- purpose solutions in an in vitro lens system
}

\section{O Matthew Oriowo}

Department of Optometry, College of Applied Medical Sciences, King Saud University, PO Box 10219, Riyadh, 11433 Saudi Arabia

<matoriowo@yahoo.com>

Received 2 November 2008; revised version accepted 5 March 2009

\begin{abstract}
Purpose: To investigate the relative cytotoxic effects of contact lens multipurpose solutions on cultured crystalline lenses.

Methods: A comparison of the fluorescence emission levels of cultured bovine lenses as affected by three hour experimental exposure to three contact lens multipurpose solutions (COMPLETE MoisturePlus, AMO; OPTI-FREE Express, Alcon; and ReNu MultiPlus, Bausch \& Lomb) was carried out. The pre- and post-exposure fluorescence levels of the lenses were obtained and values were compared to baseline and control measurements.

Results: The solutions yielded varying degrees of cytotoxicity, demonstrating significant $(p<0.01)$ reversible reduction of cellular viability levels of the cultured crystalline lenses as revealed by the degree of fluorescence emissions in the following
\end{abstract}

order (OPTI-FREE Express > ReNu MultiPlus > COMPLETE MoisturePlus multi-purpose solutions).

Conclusions: The results show that OPTI-FREE Express and ReNu MultiPlus solutions exhibited more cytotoxic effect compared to COMPLETE MoisturePlus solution. The findings support reports from previous clinical and laboratory studies. These results suggest that the in vitro approach herein presented would be a valuable system for relatively inexpensive and repeatable laboratory investigations of the possible ocular surface reactions of ophthalmic solutions, cosmetics and pharmaceuticals at pre- and during commercial phases.

Keywords: Contact lens multipurpose solutions; ocular lens; cell viability; cytotoxicity; AlamarBlue; biochemical assay; fluorescence.

\section{Introduction}

The need for contact lens practitioners to have a basic understanding of the components and the temporary ocular surface reactions from using contact lens care solutions cannot be overemphasised. This will arm the practitioner with useful knowledge with respect to what to tell patients regarding the transient ocular irritation that is possible from the use of multipurpose contact lens solutions. Currently, the most common products for disinfecting contact lenses are 
multi-purpose solutions (MPS) that can be used to clean, disinfect and wet contact lenses. Such a solution must be potent enough to kill microbial pathogens that may be harboured on the contact lenses yet must also be particularly gentle to the eye. A recent study found that contact lens wear failure was related to the product or practitioner factor rather than patient-specific problems ${ }^{1}$, implying that practitioners should possess adequate knowledge and be able to advise patients on the relative ocular surface reactions to different contact lens solutions.

It is a common view among eye care providers that efficacy against pathogenic microbes cannot be compromised in an attempt to produce an irritancyfree contact lens care solution since contact lens wear and contaminated contact lens solutions are the main causes of microbial keratitis ${ }^{2}$. For example clinical and laboratory studies have observed that the COMPLETE® multi-purpose solution has minimal toxicity compared to OPTI-FREE and ReNu solutions ${ }^{3,4}$. The fact that a solution is more comfortable, that is, has minimal cytotoxic or sensitivity effects does not guarantee full efficacy against opportunistic pathogens. For instance, recent results from two independent epidemiologic studies ${ }^{5,6}$, found that $\sim 50 \%$ - 55\% of Acanthamoeba keratitis (AK) cases used COMPLETE MoisturePlus multi-purpose solution, resulting in a more than 15 -fold increase in the risk of AK with COMPLETE MoisturePlus solution use. This led the manufacturer, Advanced Medical Optics (AMO) to voluntarily recall the COMPLETE MoisturePlus multi-purpose solution?

Contact lens multipurpose solutions (MPS) have different compositions as indicated in Table 1.

Table 1. Composition of the various multipurpose solutions.

\begin{tabular}{|l|l|l|l|l|}
\hline \multicolumn{1}{|c|}{ Solutions } & \multicolumn{1}{|c|}{ Preservatives } & Surfactant/cleaner & \multicolumn{1}{c|}{ Buffer } & \multicolumn{1}{|c|}{$\begin{array}{l}\text { Other components } \\
\text { (e.g. Electrolyte) }\end{array}$} \\
\hline $\begin{array}{l}\text { *COMPLETE } \\
\text { Moisture Plus }\end{array}$ & $\begin{array}{l}\text { 0.0001\% polyhexam- } \\
\text { ethylene biguanide } \\
\text { (PHMB) }\end{array}$ & Poloxamer 237 & $\begin{array}{l}\text { Sodium phos- } \\
\text { phate }\end{array}$ & $\begin{array}{l}\text { Potasium chloride, } \\
\text { NaCl, EDTA (0.01\%), } \\
\text { Taurine, Propylene } \\
\text { Glycol. }\end{array}$ \\
\hline ReNu MultiPlus & $\begin{array}{l}\text { p.0001\% polyaminopro- } \\
\text { pyl biguanide (PAPB) }\end{array}$ & $\begin{array}{l}\text { Poloxamine, hy- } \\
\text { droxyalkyl phospho- } \\
\text { nate }\end{array}$ & $\begin{array}{l}\text { Sodium bo- } \\
\text { rate/boric acid }\end{array}$ & EDTA (0.1\%), NaCl \\
\hline $\begin{array}{l}\text { OPTI-FREE } \\
\text { Express }\end{array}$ & $\begin{array}{l}\text { 0.001\% polidronium } \\
\text { chloride, 0.0005\% myr- } \\
\text { istamidopropyl dimeth- } \\
\text { ylamine (MAPD) }\end{array}$ & $\begin{array}{l}\text { AMP-95, Tetronic } \\
1304\end{array}$ & $\begin{array}{l}\text { Sodium } \\
\text { citrate/Boric } \\
\text { acid }\end{array}$ & $\begin{array}{l}\text { Sodium chloride } \\
\text { (NaCl), sorbital, Ede- } \\
\text { tate disodium (EDTA, } \\
\text { 0.5\%) }\end{array}$ \\
\hline
\end{tabular}

* The COMPLETE ${ }^{\circledR}$ Moisture Plus ${ }^{\mathrm{TM}}$ multi-purpose solution has lubricant/conditioner called Hydroxypropyl methyl cellulose (HPMC) in its formulation.

Manufacturers indicate that the preservatives (that is, antimicrobial agents) and other additives in the solutions have high molecular weight materials which should not normally penetrate the contact lens matrix. Hence preventing any build up to toxic levels ${ }^{8,10}$. It has been a concern that MPS preserved with even low percentages of antimicrobial agents could cause ocular surface irritation. Within the last twenty years, a number of no-rub MPS have been introduced ${ }^{4}$, 1013. MPSs are convenient and simple to use, but may present a compromise of the cleaning and disinfecting functions ${ }^{13-15}$. The reason for non-compliance among contact lens wearers is multifactorial and well documented in the literature ${ }^{16-20}$. The notion that ocular surface sensitivity to MPS may contribute to noncompliance and contact lens dropout is still controver$\mathrm{sial}^{3,21}$. The manufacturers' efforts to provide simplified care systems may lead practitioners and patients into a false sense of security and the use of disposable or frequent replacement lenses may cause them to place less emphasis on the cleaning of lenses ${ }^{22}$. The incidence and morbidity of contact lens-related mi- 
crobial keratitis have shown little change compared with reports in the late 1980 's $s^{6,23,24}$. Despite changes or claimed improvements to contact lens care solutions, microbial keratitis is still a concern in contact lens wear today, particularly in extended contact lens wear $^{24,25}$. Thus, contact lens care solutions must be efficacious against any pathogenic expressions by the microbial flora in the ocular surface.

Since contact lens MPS(s) have varying levels of antimicrobial, cleaning and lubricating activities ${ }^{26-29}$, they will inevitably present some variations in their level of ocular surface sensitivity. The OPTI-FREE Express MPS has been found to show the highest antibacterial activity against Pseudomonas aeruginosa compared to $\mathrm{ReNu}$ and COMPLETE solutions ${ }^{29}$, which should suggest more ocular surface cytotoxic or cytosensitive effect. However, a recent study reported that ReNu MultiPlus showed a more significant adverse ocular surface sensitivity effect compared to OPTI-FREE and COMPLETE solutions ${ }^{3}$. Hence, the objective of the present study was to investigate the relative cytotoxicity of three commonly used contact lens multipurpose solutions (COMPLETE moistureplus, OPTI-FREE Express and ReNu MultiPlus) to bovine lenses, using an in vitro approach. This in vitro approach employing the Alamar Blue ${ }^{\mathrm{TM}}$ biochemical assay with cultured ocular lens was recently introduced $^{30-32}$. A repeatable in vitro approach to perform comparative sensitivity evaluations among contact lens solutions would be essential, particularly when considering cost effectiveness, the need for rapid screening information and to avoid the traditional large variation that occurs with in vivo studies such as the Draize test using rabbits ${ }^{16,17,19,33-36}$. The Alamar Blue ${ }^{\mathrm{TM}}$ bioassay method utilizes the fluorescence emission levels of cultured whole crystalline lens tissue, as measured with a fluorescence multi-plate reader. The Alamar Blue ${ }^{\mathrm{TM}}$ assay model has shown consistent repeatability in its ability to detect subtle cytotoxic changes in ocular lens culture and human conjunctival cell lines ${ }^{4,30-32}$. The use of bovine crystalline lens is relevant practically and experimentally. The crystalline lens was chosen as an ocular tissue model for studying ocular tissue irritancy because; (1) The epithelium of the cornea and the lens have the same embryologic origin and are physiologically similar. (2) The main function of both structures is to focus an image on the retina. (3) The structural adap- tations of both tissues are designed to minimize light scatter, and (4) both the lens and cornea are avascu$\operatorname{lar}^{32,36}$.

\section{Methods}

\section{Multipurpose contact lens solutions}

Fourteen bottles of each of the three multipurpose solutions: ReNu MultiPlus (Bausch \& Lomb, Rochester, NY, USA); OPTI-FREE Express (Alcon Laboratories, Fort Worth, TX, USA); and COMPLETE moistureplus (Advanced Medical Optics, Dublin, Ireland) were randomly purchased from commercial retail pharmacy stores as encountered by the public. The composition of the solutions is as shown in Table 1.

\section{Crystalline Lens culture}

All culture ingredients were purchased from the Sigma Chemical Co. (St. Louis, MO, USA) unless otherwise stated. Whole crystalline lenses were excised under aseptic conditions from abattoir-obtained bovine eyes and placed in a custom 25-ml two-chambered container. The bovine eyes, from two year old cattle stock were obtained within 1-2 hour post-mortem, and held at room temperature until the dissection of the lenses, which occurred within 2 to 5 hour postmortem. To isolate the lens, the posterior portion of the eyeball was aseptically dissected, the suspensory ligaments of the lens were cut, and the adhering vitreous removed. The lenses were immersed in M199 culture medium with 3\% fetal bovine serum and $1 \%$ antibiotics (penicillin/streptomycin 100 units per ml) with sodium bicarbonate and HEPES as buffers for $\mathrm{pH}$ stability. The cultured lenses were incubated at $37^{\circ} \mathrm{C}$ in an air, $5 \% \mathrm{CO} 2$ atmosphere when not undergoing experimental measurements.

Exposure of crystalline lenses to contact lens multipurpose (MPS) solutions

The dissected lenses were kept in culture medium for 48 hours to allow for adaptation of the lenses to the medium. After the 48 hours of placing the lenses in culture medium, 40 out of 55 excised lenses were randomly allotted to treatment (utilizing 10 lenses per each experimental solution) group, and 10 lenses for the control group. The same set of 10 lenses was utilized as control for the three experimental solution groups. Fifteen lenses were not included in the study due to physical damage during dissection. The 30 
lenses selected (10 for each MPS solution treatment) for exposure were completely submerged in the three experimental solutions, respectively, for three hours at room temperature under the biological flow hood. The experimental exposure solutions comprised undiluted contact lens MPS, poured directly from the dispensing bottles into the culture chamber, each containing one lens. Untreated control lenses remained in the culture medium for three hours at room temperature under the flow hood. All procedures were done under sterile conditions utilizing a biohazard approved biological flow hood. After the three hour exposure period to MPS, the treated lenses were rinsed and the MPS replaced with fresh culture medium, and all lenses were returned to the incubator.

\section{Biochemical assay and fluorescence measurements}

Baseline fluorescence measurements were obtained for control and treated lenses prior to exposure, and at the $6,12,24,48$, and 96 hour time intervals post the 3-hour experimental exposure to contact lens MPS. Following each measurement session, all lenses were rinsed and returned to fresh culture medium. The biochemical assay system consisted of the Alamar Blue $^{\mathrm{TM}}$ assay, 12 multiwell plates, and a fluorescence plate reader. The system quantifies and records the fluorescence intensity levels of cultured ocular lenses. The fluorescence measurement is based on the principle that as radiant light wavelength is absorbed by a substance (in this case, the crystalline lens immersed in Alamar Blue ${ }^{\mathrm{TM}}$ assay) with the excitation wavelength of $530 \mathrm{~nm}$, a certain amount of fluorescence emission will result at a longer wavelength (in this case at $590 \mathrm{~nm}$ ). The Alamar Blue $\mathrm{TM}^{\mathrm{TM}}$ dye (obtained from MEDICORP Inc., Montreal, Canada) is used to quantify the viability level of living cells in vitro ${ }^{37}$. It incorporates resazurin and resorufin as a fluorometric-colorimetric oxidation-reduction (Redox) indicator that fluoresces and changes colour in response to reduction resulting from cell metabolism ${ }^{37,38}$. It has been reported that serum interferes with Alamar Blue ${ }^{\mathrm{TM}}$ fluorescence readings by inducing reduction of the dye ${ }^{39}$, therefore it was ensured that the experimental assay medium was serum free.

The Alamar Blue ${ }^{\mathrm{TM}}$ dye was diluted into the culture medium (modified M199) without serum, to 8\% (v/v). The assay solution was prepared immediately before use at each measurement session using a 100 $\mu 1$ Eppendorf ${ }^{\circledR}$ "Tip-Ejector" microlitre pipette.
Freshly prepared assay solution before each use at every measurement time point was to avoid possible precipitation of the assay dye. Both the treated and control lenses were transferred into sterile 12-well flat bottom tissue culture plates (Costar, Cambridge, MA, USA) with one lens per well. The culture medium containing serum was carefully aspirated, and the lenses rinsed twice with 6-ml of experimental medium with no serum. Then $3.8-\mathrm{ml}$ of the assay solution was added to each well containing a lens, using a sterile 250- $\mu 1$ adjustable pipette tip and an Eppendorf repeater pipette. A prior pilot study by the present investigator showed a two hour incubation period to be optimum for the assay to diffuse into the lenses for fluorescence measurement. Therefore at each measurement session, both control and experimental lenses were incubated for two hours in the assay solution, after which the fluorescence measurements were performed with a CytoFluor ${ }^{\mathrm{TM}}$ II fluorescence multiwell plate reader (PerSeptive Biosystems Inc., Framingham, MA, USA). Thirty minutes prior to performing the fluorescence measurements, the excitation / emission wavelength settings on the CytoFluor ${ }^{\mathrm{TM}}$ plate reader were adjusted to $530 / 590 \mathrm{~nm}$ with the sensitivity gain set at 50 , and temperature at $37^{\circ} \mathrm{C}$. The plate reader probe was set to scan 10 different positions in each lens. Thus, an average of 10 readings was obtained for every lens. Therefore, each fluorescence level data represent an average of 10 readings per scan for each lens at least six times through the study duration, amounting to a total of 2400 quantitative lens fluorescence intensity measures.

\section{Statistical analysis}

Results were expressed as the mean \pm the standard deviation (S.D) of the mean. The data were analysed using the paired $t$-test and repeated-measures analysis of variance (ANOVA). Repeated measures ANOVA was used to compare the control group with the treated groups of lenses across all six measurement time periods. A repeated measures ANOVA found a significantly interaction between time and group $(p<$ 0.001 ). For within group analysis, the baseline (that is, pre-exposure) and follow up post-exposure fluorescence readings of lenses in the same group at the predetermined intervals were compared. The data were also compared between groups at each time point. The Dunnett multiple statistical test was used for within group comparisons (comparing baseline to 
subsequent measurements in the same group), and the Tukey-Kramer multiple comparisons test for between group comparison at baseline and the respective follow-up time points. Any probability $p$ value less than 0.01 was considered significant. The extent of cytotoxicity is judged by each $p$ value which indicates the degree of significant effects as follows: $p$ values equal or less than 0.01 (significant) $; \leq 0.001>0.0001$ (highly significant); and $\leq 0.0001>0.00001$ (extremely significant). The total number of times each solution exhibited minimal to extremely significant effect will be used to determine and discuss the relative rank order of cytotoxicity among the solutions.

\section{Results}

The results for the different relative cytotoxic effects of the solutions are shown in Tables 2 to 4 as the mean $( \pm$ S.D $)$. At 6 hours, lenses treated with OPTIFREE and ReNu (Tables 2 and 3) demonstrated significant cytosensitive effects with $p$ values of 0.001 and 0.01 , respectively. The control lenses $(n=10)$, and lenses treated with COMPLETE solution $(n=$ 10) did not show significant changes in fluorescence profiles throughout the duration of the experiment (Table 4). The OPTI-FREE treated lenses exhibited a significant recovery at $12 \mathrm{hr}$, but with a rebounce at $24 \mathrm{hr}(p=0.00001)$ and eventually back to recovery by $96 \mathrm{hr}$. The fluorescence levels of ReNu treated lenses did not show a recovery at $12 \mathrm{hr}(p=0.012)$, but exhibited gradual recovery beginning from $24 \mathrm{hr}$ to baseline and control levels by the end of the $96 \mathrm{hr}$ study (Table 3).

In order to rank the order of cytotoxicity among the three solutions, it was considered that the level of significance at each time point would indicate the degree of cytotoxic effect of each solution. Therefore, judging from the $p$ values for each solution at different intervals as shown in Tables 2 - 4, the descending rank order of the cytotoxic effect is as follows: OPTIFREE $>$ ReNu $>$ COMPLETE solutions, with OPTIFREE showing the most cytotoxicity effect.

Table 2. Descriptive statistics of the fluorescence data for lenses exposed to OPTI-FREE multipurpose contact lens solution $(n=$ 10 for exposed lenses, and $n=10$ for control lenses).

\begin{tabular}{|c|c|c|c|c|c|}
\hline \multirow[t]{2}{*}{$\begin{array}{l}\text { Measurement time } \\
\text { (hr) }\end{array}$} & \multicolumn{2}{|c|}{$\begin{array}{l}\text { Fluorescence level } \\
\text { (mean } \pm \text { S.D.) }\end{array}$} & \multicolumn{2}{|c|}{$\begin{array}{l}p \text { values (within group) } \\
\text { Contrast to baseline }\end{array}$} & \multirow{2}{*}{$\begin{array}{c}p \text { values } \\
\text { (Between groups) } \\
\text { Exposed versus Control }\end{array}$} \\
\hline & Exposed & Control & Exposed & Control & \\
\hline Baseline & $34656 \pm 2855$ & $34835 \pm 3811$ & & & 0.907 \\
\hline 6 & $28007 \pm 3866$ & $37042 \pm 3379$ & 0.001 & 0.10 & 0.00003 \\
\hline 12 & $33820 \pm 3287$ & $35692 \pm 4192$ & 0.574 & 0.62 & 0.281 \\
\hline 24 & $27751 \pm 3132$ & $35431 \pm 2360$ & 0.00001 & 0.71 & 0.00002 \\
\hline 48 & $27007 \pm 2819$ & $33647 \pm 2834$ & 0.00001 & 0.13 & 0.00005 \\
\hline 96 & $32952 \pm 3651$ & $35725 \pm 4585$ & 0.245 & 0.60 & 0.153 \\
\hline
\end{tabular}

Note: Baseline readings were taken before exposing lenses to MPS. The same set of control lenses were utilised for OPTI-FREE, Complete, and ReNu solution exposures. Values are presented as arbitrary fluorescent units. Grading the level of cytotoxicity at different time points: $p=0.01$ (minimal), 0.001 (very adverse), $<0.0001$ (extremely adverse).

Table 3. Descriptive statistics of the fluorescence data for lenses exposed to ReNu multipurpose contact lens solution $(n=10$ for exposed lenses, and $n=10$ for control lenses).

\begin{tabular}{|l|l|l|l|l|l|}
\hline \multirow{3}{*}{$\begin{array}{c}\text { Measurement time } \\
(\mathrm{hr})\end{array}$} & \multicolumn{2}{|c|}{$\begin{array}{c}\text { Fluorescence level } \\
\text { (mean } \pm \text { S.D.) }\end{array}$} & \multicolumn{2}{c|}{$\begin{array}{c}p \text { values (within group) } \\
\text { Contrast to baseline }\end{array}$} & $\begin{array}{c}p \text { values } \\
\text { (Between groups) } \\
\text { Exposed versus Control }\end{array}$ \\
\cline { 2 - 5 } & Exposed & Control & Exposed & Control & \\
\hline Baseline & $34720 \pm 3313$ & $34835 \pm 3811$ & & & 0.944 \\
\hline 6 & $28392 \pm 5295$ & $37042 \pm 3379$ & 0.005 & 0.10 & 0.001 \\
\hline 12 & $28141 \pm 5745$ & $35692 \pm 4192$ & 0.012 & 0.62 & 0.002 \\
\hline 24 & $31161 \pm 2887$ & $35431 \pm 2360$ & 0.016 & 0.71 & 0.002 \\
\hline 48 & $35088 \pm 2973$ & $33647 \pm 2834$ & 0.820 & 0.13 & 0.282 \\
\hline 96 & $35157 \pm 4166$ & $35725 \pm 4585$ & 0.585 & 0.60 & 0.775 \\
\hline
\end{tabular}


Table 4. Descriptive statistics of the fluorescence data for lenses exposed to COMPLETE multipurpose contact lens solution ( $n=$ 10 for exposed lenses, and $n=10$ for control lenses).

\begin{tabular}{|l|l|l|l|l|l|}
\hline \multirow{2}{*}{$\begin{array}{c}\text { Measurement time } \\
\text { (hr) }\end{array}$} & \multicolumn{2}{|c|}{$\begin{array}{c}\text { Fluorescence level } \\
\text { (mean }\end{array}$} & \multicolumn{2}{c|}{$\begin{array}{c}\text { values (within group) } \\
\text { Contrast to baseline }\end{array}$} & \multicolumn{1}{c|}{$\begin{array}{c}p \text { values } \\
\text { (Between groups) } \\
\text { Exposed versus Control }\end{array}$} \\
\cline { 2 - 5 } & Exposed & Control & Exposed & Control & \\
\hline Baseline & $35599 \pm 3881$ & $34835 \pm 3811$ & & & 0.66 \\
\hline 6 & $36381 \pm 2389$ & $37042 \pm 3379$ & 0.55 & 0.10 & 0.62 \\
\hline 12 & $35537 \pm 2763$ & $35692 \pm 4192$ & 0.96 & 0.62 & 0.92 \\
\hline 24 & $34470 \pm 1903$ & $35431 \pm 2360$ & 0.42 & 0.71 & 0.33 \\
\hline 48 & $35205 \pm 1727$ & $33647 \pm 2834$ & 0.70 & 0.13 & 0.16 \\
\hline 96 & $35744 \pm 3658$ & $35725 \pm 4585$ & 0.91 & 0.60 & 0.99 \\
\hline
\end{tabular}

\section{Discussion}

The fact that the last decade has witnessed contact lens wearers changing to disposable and frequent replacement soft lenses with multipurpose solutions requires the contact lens practitioner to have informed knowledge on the efficacy and relative irritancy of multipurpose contact lens care regimens ${ }^{3,10,25}$. Contact lens multipurpose solutions contain quaternary ammoniums or polymers of hexamethylene biguanide (PHMB) as active preservative agents. Preservatives are widely used in agricultural and food chemistry to keep food fresh, and in the cosmetic industry to avoid spoilage or chemical changes by microbes such as bacteria and fungi. Preservatives are also commonly used as sanitizers for baby wipes, pool and spa, and as disinfection products in medical preparations such as eye drops or contact lens solutions ${ }^{4}$.

Multipurpose solutions are classified as medical devices (class 2b) and can impregnate the contact lens during the soaking time and insertion on ocular surface. Chemicals including preservatives contained in contact lens solutions could initiate ocular surface cytotoxic reactions or contact lens intolerance. The findings of the present study show that the three contact lens MPSs induced varying levels of reversible lens cytotoxic reactions, with OPTI-FREE Express No Rub ${ }^{\circledR}$ solution showing the most effect. Similar observations on cultured human conjunctival cell lines showed that OPTI-FREE Express solution was significantly more toxic than $\mathrm{ReNu}$ MultiPlus No Rub ${ }^{\circledR}$ and COMPLETE moistureplus ${ }^{\mathrm{TM}}$ solution, respectively ${ }^{4}$. In contrast, an in vivo observation from a recent clinical study found that $\mathrm{ReNu}$ MultiPlus produced the most adverse ocular surface effect compared to OPTI-FREE and COMPLETE solutions ${ }^{3}$.

From the findings in the present study it might be assumed that the COMPLETE moistureplus ${ }^{\mathrm{TM}}$ MPS would have relatively little sensitivity effect compared to OPTI-FREE or ReNu. However, there is a caveat in the assumption in that the present study is an exploratory in vitro investigation as opposed to in vivo experiment in which actual ocular surface irritancy effects can be directly obtained. A future investigation is required to conduct a parallel study of in vitro and in vivo evaluation of sensitivity effects of contact lens MPSs, and study the relationship between ocular lens cytotoxicity findings and actual ocular surface sensitivity effects. One possible explanation for the difference in the MPS cytotoxic effects is that OPTI-FREE solution has a completely different antimicrobial agent $(0.0005 \%$ myristamidopropyl dimethylamine (MAPD), while ReNu and COMPLETE moistureplus MPSs are both $0.0001 \%$ of polyhexamethylene biguanide (PHMB)-preservative based solution systems. The antimicrobial action of MAPD is not fully known but has been proposed as similar to the action of PHMB and chlorhexidine which causes cytoplasmic membrane damage leading to loss of essential cellular components following binding to the cell wall ${ }^{40}$. Also, as shown in Table 1, the COMPLETE solution has hydroxypropyl methylcellulose (HPMC) as lubricant in its formulations compared to ReNu and OPTIFREE solutions. The findings in the present study indicate that the chemical variations which exist between the solutions would yield differential cytotoxic reactions. However, the results show that there will be 
recovery from such irritancy or cytotoxic effect from the solutions.

In the field of toxicology, the Draize ${ }^{41}$ test has been the standard in vivo measure of ocular toxicity for over fifty years. It is based on observations of irritation and injury to the cornea, conjunctiva, and iris after the application of test chemicals to the eyes of live rabbits 33,34 . However, because of poor sensitivity and repeatability and ethical concerns about the suffering of live animals, there is an increasing need for more in vitro alternatives ${ }^{33,34,36}$, and researchers have continued to develop more in vitro approaches for determining ocular toxicity ${ }^{30,36,42}$. Crystalline lens culture has increasingly been used for in vitro alternative methods in ocular toxicology $30,32,36,42$. Unlike the cornea, the ocular lens can be cultured as an intact organ for long periods as it can retain its physiological integrity during the culture period, particularly with its repair mechanisms preserved. As earlier mentioned the crystalline lens has a number of similarities to the cornea to support its use as a model for corneal irritancy testing. It is an avascular tissue and its principal function is to transmit light to the retina. Both the lens and cornea have structural and physiological adaptations to refract light.

According to the findings in the present study, there is no indication that any of these solutions would result in permanent adverse cytotoxic damage to the ocular surface tissue with clinical or patient care use. Concerning the trade-off between relative efficacy and comfort, the findings of relative cytotoxic effect between the three solutions as demonstrated in the present study appear to agree with the findings of Leung et al. ${ }^{29}$, who found that OPTIFREE Express showed the highest antimicrobial activity against Pseudomonas aeruginosa compared to $\mathrm{ReNu}, \mathrm{COMPLETE}$ and Solo-care solutions at $4{ }^{\circ} \mathrm{C}$, $25^{\circ} \mathrm{C}$ and $30^{\circ} \mathrm{C}$, however this is not the focus of the present study. An ideal contact lens MPS would have both low cytotoxic effect and high antimicrobial efficacy. Efficacy will always be of paramount importance in contact lens multipurpose solutions because it has been found that even a standard contact lens care hygiene regime does not seem to be sufficient in preventing the development of corneal infection and ulcers in contact lens (particularly in conventional and frequent replacement daily wear soft contact lenses) wearers ${ }^{25,43}$. In terms of in vitro methodology, the Alamar Blue ${ }^{\mathrm{TM}}$ biochemical assay has been used in many fields, especially in pharmacology to screen for agents toxic to mammalian cells ${ }^{44}$. The results in the present study agree with the previous clinical/laboratory findings ${ }^{3,45}$, and those of Pharm and Huff 46 who used the Alamar Blue ${ }^{\mathrm{TM}}$ assay to study cytotoxic effect of contact lens solutions on bovine corneal epithelial cultures.

In conclusion, these results confirm that OPTI-FREE is more cytotoxic compared to $\mathrm{ReNu}$ and COMPLETE contact lens multipurpose solutions. The in vitro system herein presented offers a quick and quantitative in vitro assessment of the efficacy and potential irritability of contact lens solutions. As well, it would be a valuable system for relatively inexpensive and repeatable laboratory investigations of the possible ocular surface reactions of ophthalmic solutions, cosmetics and pharmaceuticals at pre- and during commercial phases.

\section{Declaration:}

The author has no proprietary or commercial interest in the products named in this article.

\section{References}

1. Young G, Veys J, Pritchard N, Coleman S. A multi-centre study of lapsed contact lens wearers. Ophthal Physiol Opt 200222 516-527.

2. Dart JK, Stapleton F, Minassian D. Contact lenses and other risk factors in microbial keratitis. Lancet 1991338 650-653.

3. Lievens, C.W. Hakim, N., Chinn, A. The effect of multipurpose solutions on the ocular surface. Eye Contact Lens 200632 8-11.

4. Dutot M, Warnet JM, Baudouin C, Rat P. Cytotoxicity of contact lens multipurpose solutions: Role of oxidative stress, mitochondrial activity and P2X7 cell death receptor activation. Euro J Pharmaceutical Sciences 200833 138145.

5. Centers for Disease Control and Prevention. Acanthamoeba keratitis multiple states, 2005-2007. MMWR Morb Mortal Wkly Rep 200756 532-534.

6. Joslin C, Tu EY, Shoff ME, Booton GC, Fuerst PA, McMahon TT, Anderson RJ, Dworkin MS, Sugar J, Davis FG, Stayner LT. The association of contact lens solution use and Acanthamoeba keratitis. Am J Ophthalmol 2007144 169180.

7. U.S. Food and Drug Administration. Advanced medical optics voluntarily recalls Complete ${ }^{\circledR}$ MositurePlus ${ }^{\mathrm{TM}}$ contact lens solution. Available at http://www.fda.gov/oc/po/firmrecalls/amo0507.html. Accessed on Oct 18, 2007.

8. Lowe R, Vallas V, Brennan N. Comparative efficacy of contact lens disinfection solutions. CLAO 199218 34-40. 
9. McLaughlin R. Rub versus no rub: looking at MPS care solutions. Contact Lens Spectrum 200116 40-45.

10. Rakow PL. Current contact lens care systems. Ophthalmol Clin North Am 200316 415-432.

11. Guillon M, Maissa C. Clinical acceptance of two multipurpose solutions: MPS containing HPMC versus citrate-based MPS without rubbing. CLAO 200228 186-191.

12. McGrath D, Costanzo SP, Manchester RJ, Kaiser JJ, Norton SE, McCormick PJ. Comparative antimicrobial activity of no-rub multipurpose lens care solutions in the presence of organic soil. Eye Contact Lens 200529 245-249.

13. Borazjani RN, Kilvington S. Effect of a multipurpose contact lens solution on the survival and binding of Acanthamoeba species on contact lenses examined with a no-rub regimen. Eye Contact Lens 200531 39-45.

14. Greco A. All-purpose solutions: compromise versus compliance. International Eyecare 1985 1356-358.

15. Liedel KK, Begley CG. The effectiveness of soft contact lens disinfection systems against Acanthamoeba on the lens surface. J Am Optom Assoc 199667 135-142.

16. Chun M, Weissman B. Compliance in contact lens care. Am J Optom Physiol Opt 1986164 274-276.

17. Collins M, Carney L. Patient compliance and its influence on contact lens wearing problems. Am J Optom Physiol Opt 198663 952-956.

18. Sokol JL, Mier MG, Bloom S, Asbell PA. A study of patient compliance in a contact lens wearing population. CLAO 199016 209-213.

19. Claydon B, Efron N. Non compliance in contact lens wear. Ophthal Physiol Opt 199414 356-364.

20. O’Donnell C, Efron N. Non-compliance with lens care and maintenance in diabetic contact lens wearers. Ophthal Physiol Opt 200424 504-510.

21. Donshik P, Madden R, Simmons PA. Pursuing comfort in a multipurpose solution. Contact Lens Spectrum 200014 33-36.

22. Mowrey-McKee M, Sills A, Wright A. CIBA Vision Corporation. Comparative cytotoxicity potential of soft contact lens care regimens. CLAO 200228 160-164.

23. Chen KH, Leung SL, Hoekman HW, Beekhuis WH, Mulder PGH, Geerards AJM, Kijlstra A. Incidence of contact-lensassociated microbial keratitis and its related morbidity. Lancet 1999354 181-185.

24. Buck SL, Rosenthal RA, Schlech BA. Amoebicidal activity of multipurpose contact lens solutions. Eye Contact Lens J 200531 62-66.

25. Shoff ME, Joslin CE, Tu EY, Kubatko L, Fuerst PA. Efficacy of contact lens systems against recent clinical and tap water Acanthamoeba isolates. Cornea 200827 713-719.

26. Thai LC, Tomlinson A, Simmons PA. In vitro and in vivo effects of a lubricant in a contact lens solution. Ophthal Physiol Opt 200222 319-329.

27. Beattie TK, Seal DV, Tomlinson A, McFadyen AK, Grimason AM. Determination of amoebicidal activities of multipurpose contact lens solutions by using a most probable number enumeration technique. J Clin Microbiol 200341 2992-3000.

28. Codling CE, Maillard JY, Russell AD. Performance of contact lens disinfecting solutions against Pseudomonas aeruginosa in the presence of organic load. Eye Contact Lens $J$
200329 100-102.

29. Leung P, Boost MV, Cho P. Effect of storage temperatures and time on the efficacy of multipurpose solutions for contact lenses. Ophthal Physiol Opt 200424 218-224.

30. Oriowo OM, Cullen AP, Schirmer K, Chou BR, Bols NC, Sivak JG. Evaluation of porcine lens and a fluorescence assay approach for in vitro ocular toxicological investigations. ATLA J 200230 505-513.

31. Oriowo OM. Alamar Blue bioassay for in vitro cellular investigation of UV-crystalline lens damage. Ophthal Physiol Opt 200323 307-314.

32. Youn HY, Moran KL, Oriowo OM, Bols NC, Sivak JG. Surfactant and UV-B-induced damage of the cultured bovine lens. Toxicol In Vitro 200418 841-852.

33. Weil CS, Scala RA. Study of intra- and interlaboratory variability in the results of rabbits eye and skin irritation tests. Toxicol Appl Pharmacol 197119 276-360.

34. Koch W. Validation criteria for ocular irritation in vitro alternative tests. J Toxicol: Cutaneous and Ocular Toxicology 19898 17-22.

35. Gower L, Stein J, Turner F. Compliance: a comparison of three lens care systems. Optom Vis Sci 199471 629-643.

36. Sivak JG, Herbert KL, Fonn D. In vitro ocular irritancy measure of four contact lens solutions: damage and recovery. CLAO 199521 169-74.

37. O’Brien J, Wilson I, Orton T, Pognan F. Investigation of the Alamar Blue (resazurin) fluorescent dye for the assessment of mammalian cell cytotoxicity. Euro J Biochem 2000267 5421-5426.

38. Larson EM, Doughman DJ, Gregerson DS, Obritsch WF. A new, simple, non-radioactive, nontoxic in vitro assay to monitor corneal endothelial cell viability. Invest Ophthalmol Vis Sci 199738 1929-1933.

39. Goegan P, Johnson G, Vincent R. Effects of serum protein and colloid on the Alamar blue assay in cell cultures. Toxicol In Vitro 19959 257-266.

40. Hughes R, Dart J, Kilvington S. Activity of the amidoamine myristamidopropyl dimethylamine. J Antimicrob Chemother 200351 1415-1418.

41. Draize JH, Woodward G, Calvery HO. Methods for study of irritation and toxicity of substances applied topically to the skin and mucous membranes. J Pharmacol Exp Ther 194482 377-390.

42. Bantseev V, McCanna D, Banh A, Wong WW, Moran KL, Dixon DG, Trevithick JR, Sivak JG. Mechanisms of ocular toxicity using the in vitro bovine lens and sodium dodecyl sulfate as a chemical model. Toxicol Sci 2003 73 98-107.

43. Najjar DM, Aktan SG, Rapuano CJ, Laibson PR, Cohen EJ. Contact lens-related corneal ulcers in compliant patients. Am J Ophthalmol 2004137 170-172.

44. Evans SM, Casartelli A, Herreros E, Minnick DT, Day C, George E, Westmoreland C. Development of a high throughput in vitro toxicity screen predictive of high acute in vivo toxic potential. Toxicol In Vitro 200115 579-584.

45. Oriowo MO. A fluorometric study of relative ocular lens cytosensitivity to multipurpose contact lens solutions using the resazurin assay method. Toxicol In Vitro 200620 1548-1554.

46. Pharm X, Huff J. Cytotoxicity evaluation of multipurpose contact lens solutions using an in vitro test battery. CLAO 199925 28-35. 